\title{
Improving post-CRT neck assessment in patients with HPV-associated OPSCC (Review)
}

\author{
MICHAEL WOTMAN $^{1}$, MAGED GHALY ${ }^{1}$, LUKE MASSARO ${ }^{1}$, TRISTAN THAM ${ }^{2}$, NAGASHREE SEETHARAMU ${ }^{3}$, \\ DEV KAMDAR $^{2}$, DOUGLAS FRANK ${ }^{2}$, DENNIS KRAUS ${ }^{2}$ and SEWIT TECKIE $^{1}$ \\ Departments of ${ }^{1}$ Radiation Medicine, ${ }^{2}$ Otolaryngology-Head and Neck Surgery and ${ }^{3}$ Medicine, \\ Donald and Barbara Zucker School of Medicine at Hofstra/Northwell, New York, NY 11549, USA
}

Received December 14, 2019; Accepted April 10, 2020

DOI: $10.3892 / \mathrm{mco} .2020 .2094$

\begin{abstract}
The positive predictive value (PPV) of 12-week post-therapy FDG-PET/CT is low in patients with Human Papillomavirus (HPV)-associated Oropharyngeal Squamous Cell Carcinoma (OPSCC) after treatment with definitive chemoradiation (CRT). Moreover, the diagnostic performance of post-CRT fine needle aspiration (FNA) in detecting persistent disease is unknown in this population. Given these important shortcomings in post-CRT treatment assessment, head and neck oncologists are limited in appropriately selecting patients for consolidative neck dissection, which results in over-treatment of a favorable risk population. Using the PubMed database, we performed a literature review of published series in HPV-associated OPSCC to investigate potential strategies for improvement of post-CRT neck assessment. Several different approaches were found, including continued surveillance with PET/CT, delayed timing of restaging PET/CT, initial response evaluation with multimodality or alternative imaging, and detection of circulating HPV DNA. At present, the optimal approach to post-CRT treatment assessment is unclear; further investigation and incorporation of new technologies and surveillance protocols will be highly beneficial for patients with HPV-associated OPSCC.
\end{abstract}

\section{Contents}

1. Introduction

2. Strategies to improve post-CRT neck assessment

3. Conclusions and prospects

Correspondence to: Dr Sewit Teckie, Department of Radiation Medicine, Donald and Barbara Zucker School of Medicine at Hofstra/Northwell, 130 East 77th Street, 1st Floor, New York, NY 10075, USA

E-mail: steckie@northwell.edu

Key words: head and neck neoplasms, papillomaviridae, oropharyngeal neoplasms, chemoradiotherapy, positron-emission tomography, neck dissection

\section{Introduction}

Current treatment assessment algorithms. Definitive chemoradiation (CRT) is a standard treatment for locally-advanced human papillomavirus (HPV)-associated oropharyngeal squamous cell carcinoma (OPSCC). The response to treatment is assessed with both clinical and radiographic information. In patients without evidence of primary or nodal disease on clinical examination, surveillance imaging is often performed with a 12-week FDG-PET/CT. Both lymph node size and intensity of FDG uptake are used to assess the likelihood of residual disease in the neck and guide the decision to either observe patients or proceed with neck dissection. Both NCCN and UpToDate have published algorithms to assist clinicians with this process $(1,2)$. NCCN recommends observation for negative scans, observation or repeat PET/CT at 3-6 months for equivocal scans, and CT or MRI followed by neck dissection for positive scans (Fig. 1). Negative scans are defined by no or low-grade uptake and positive scans are defined as suspicious for disease. According to UpToDate (Fig. 2), there are five different options depending on imaging and clinical factors. It is recommended that patients undergo salvage surgery for PET positive/CT positive scans and clinical observation for PET negative/CT negative scans. If a patient has a PET negative and CT positive result, 2-3 monthly clinical and radiographic observation is recommended for high-risk patients, while clinical observation is suggested for low-risk individuals, including those with HPV-associated OPSCC. Finally, a repeat PET/CT at 4-6 weeks is recommended for those with equivocal findings.

Low positive predictive value of 12 -week PET/CT. Although these surveillance strategies have been shown to promote excellent oncologic control, replacing the policy of planned neck dissections, they may not be broadly applicable to all head and neck cancers (3-7). In HPV-associated OPSCC, the reported positive predictive value (PPV) of 12-week post-therapy PET/CT is low (8-11), likely secondary to the lower pre-test probability of residual nodal disease (12). In a retrospective chart review of 233 patients with HPV-associated OPSCC, Corpman et al found that post-treatment PET/CT had a PPV of $13.4 \%$ and led to 60 additional imaging studies, 23 additional biopsies, and meaningful salvage therapy in only 3 patients (1.6\%) (13). One of the primary limitations of using PET/CT is the lack of a 
standardized response criteria for HPV-associated disease. It has become increasingly clear that this disease entity has unique radiographic features, including a longer time to complete lymph node involution (14). This finding has been incorporated into the UpToDate algorithm, which recommends that CT positive/PET negative scans be managed with clinical observation in HPV-associated OPSCC. However, there is currently no effective strategy to manage lymph nodes with residual FDG avidity. Current algorithms guide decision-making by dividing responses into positive and equivocal groups. However, this approach may not be appropriate in HPV-associated disease. In a study of 79 patients with HPV-associated OPSCC on a de-intensification trial, Wang et al demonstrated a low PPV for $\mathrm{PET} / \mathrm{CT}$ when responses were categorized as equivocal/incomplete or incomplete (9 and 13\%, respectively) (9). The authors used the Mehanna et al trial definitions for nodal response (3), which defined an incomplete response as 'intense FDG uptake at 12 weeks after chemoradiotherapy, with or without enlarged lymph nodes,' and equivocal response as 'mild or no FDG uptake in enlarged nodes or mild FDG uptake in normal-sized nodes.'

Limitations of Post-CRT fine-needle aspiration. While FNA has central importance in the initial workup of head and neck cancers, the utility of post-CRT FNA in detecting persistent neck disease is limited. The accuracy of post-CRT FNA in head and neck cancer has been questioned given the difficulty in interpreting the viability of irradiated cancer cells. In a series of 540 patients with cancers of the oral cavity, oropharynx, hypopharynx, and larynx, van der Van der Putten et al found that post-CRT FNA had a specificity of $42 \%$ in patients with advanced disease; only $50 \%$ of patients with positive cytology had viable disease on neck dissection pathology (15). On the other hand, in a pilot study of 14 patients undergoing FNA before planned neck dissection for persistent lymphadenopathy on imaging, Fleischman et al found that the diagnostic accuracy of FNA was $88 \%$ at detecting residual head and neck cancer (16). The authors suggested that FNA may be a feasible ancillary diagnostic modality. In truth, the diagnostic performance of post-CRT FNA has not been studied in HPV-associated OPSCC, indicating that future research is needed before clinicians can rely on this modality to reconcile concerning PET/CT findings.

Aims of this article. Given these important shortcomings in post-CRT treatment assessment in patients with HPV-associated OPSCC, physicians are limited in appropriately selecting patients for consolidative neck dissection, which results in over-treatment. This consideration is especially important for patients with HPV-associated disease, a population with a favorable prognosis. Post-CRT neck dissection has important implications on long-term quality of life as it is associated with a higher rate of post-operative complications (17). Therefore, a literature review was performed with the PubMed Database to investigate potential strategies for improvement of post-CRT treatment assessment of the neck in patients with HPV-associated OPSCC.

\section{Strategies to improve post-CRT neck assessment}

The existing literature highlights several potential strategies to improve post-CRT neck assessment in patients with
HPV-associated OPSCC (Table I), including continued surveillance with $\mathrm{PET} / \mathrm{CT}$, delayed timing of restaging PET/CT, initial assessment with multimodality or alternative imaging, and detection of circulating HPV DNA.

Continued surveillance with PET/CT. Numerous publications have promoted the notion of exercising caution when interpreting 12-week PET/CT results, adopting a high threshold for immediate neck dissection, and observing patients with serial imaging $(3,9,13)$. One study was found that systematically evaluated a surveillance strategy in patients with HPV-associated OPSCC (18). In a retrospective analysis of a prospectively collected database, Liu et al assessed the utility of a repeat PET/CT, instead of immediate neck dissection, in patients with HPV-associated OPSCC who underwent RT or CRT, and had an incomplete response on 12-week PET/CT (18). The authors enrolled 235 patients with non-metastatic disease; 41 patients underwent 16 -week imaging for an incomplete response at 12-weeks, which included both positive and equivocal responses. A positive response was defined as focal FDG uptake greater than the liver and corresponding to a structural abnormality, while an equivocal response was defined as focal FDG uptake less than the liver, greater than adjacent tissue, and corresponding to a structural abnormality. Patients were excluded from undergoing 16-week imaging if they had residual FDG avidity at the primary or distant site on 12-week PET/CT or if they had evidence of clinical nodal progression $(n=95)$. Re-evaluation at a multidisciplinary tumor board was performed for patients with a persistent incomplete response on 16 -week imaging. In their analysis, the authors found that 16 -week $\mathrm{PET} / \mathrm{CT}$ reduced the rate of neck dissection from 17 to $2.6 \%$ without impacting subsequent regional control; no regional failures occurred in patients who underwent neck dissection following a 16-week PET/CT. Moreover, 29 (71\%) patients converted to a complete response on 16-week imaging, only one of which had nodal failure at 49 months post-therapy. Compared to a PPV of $12 \%$ for 12 -week PET/CT, the PPV of 16-week imaging was $33 \%$. Based on these findings, the authors suggested that a 16-week repeat PET/CT could spare many patients from unnecessary surgeries.

There are several important considerations pertaining to this study. Regarding the inclusion criteria, the authors grouped both positive and equivocal responses as incomplete, which is appropriate given that both an equivocal and positive response pose a clinical dilemma. PET/CT scans with significant FDG avidity may still be false positives in HPV-associated disease. In regard to the exclusion criteria, patients did not undergo repeat PET/CT if they had evidence of clinical nodal progression or had residual FDG avidity at the primary or a distant site on 12-week PET/CT. These exclusion criteria are also reasonable given the increased likelihood of disease in these clinical scenarios. In a retrospective analysis of 146 patients with OPSCC and treated with CRT, Bird et al found that all patients with residual FDG-uptake at both the primary site and neck had pathologically confirmed persistent disease (19), indicating that these patients may not be appropriate for observation. Finally, it is important to consider how the authors managed patients with persistent FDG avidity on 16-week imaging. Eight patients had stable FDG avidity on 16-week 


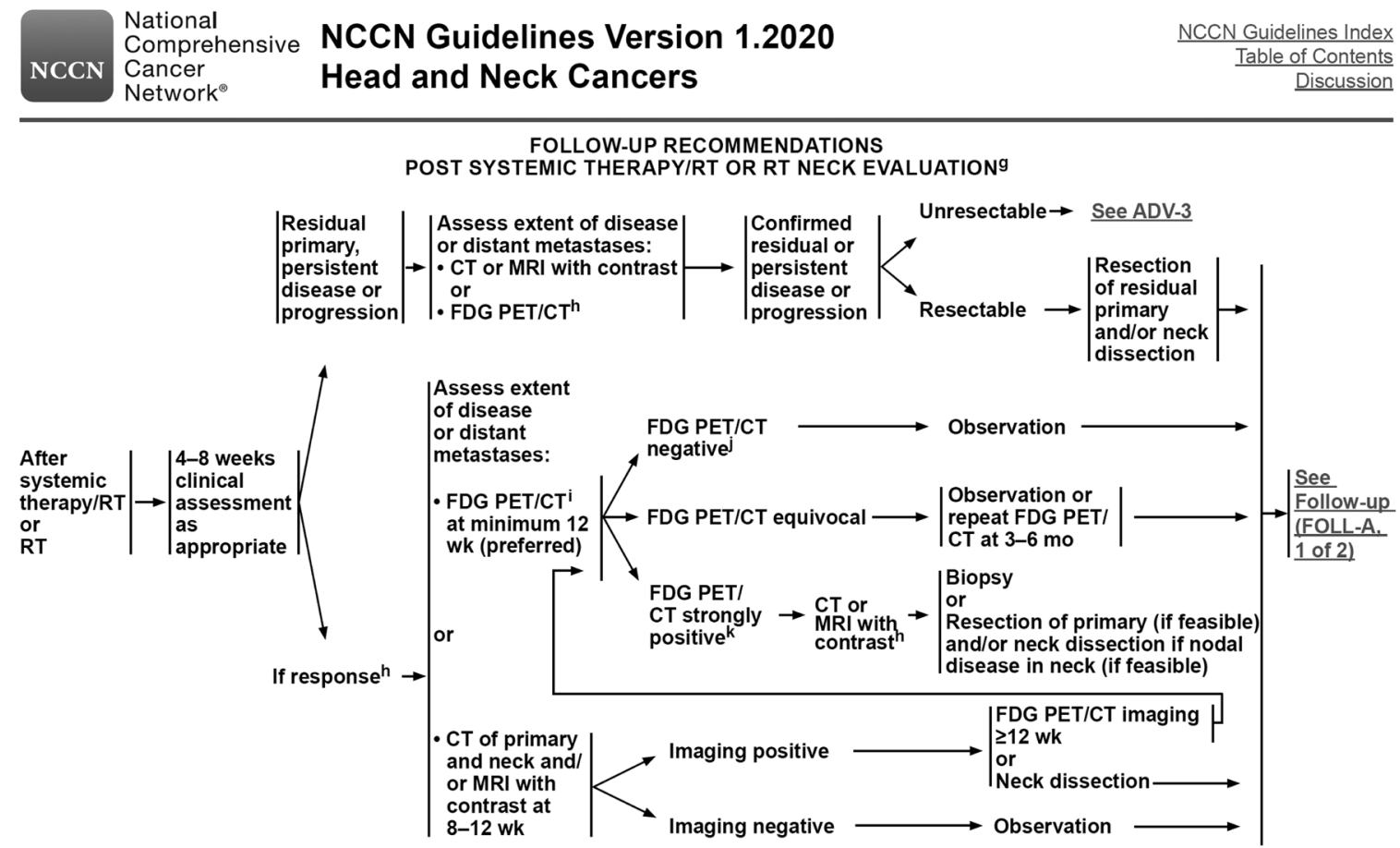

gAdapted with permission from Kutler DI, Patel SG, Shah JP. The role of neck dissection following definitive chemoradiation. Oncology 2004;18:993-998.

ilf an FDG PET/CT is performed and negative for suspicion of persistent cancer, further cross-sectional imaging is optional. JPET negative $=$ No or low-grade uptake, felt not suspicious for disease KPET positive $=$ PET suspicious for disease.

Note: All recommendations are category $2 \mathrm{~A}$ unless otherwise indicated.

Clinical Trials: NCCN believes that the best management of any patient with cancer is in a clinical trial. Participation in clinical trials is especially encouraged.
Cliction

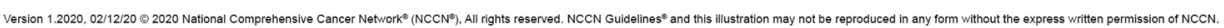

Figure 1. NCCN Follow-up Recommendations for Post Systemic Therapy/RT or RT Neck Evaluation. Reproduced with permission from the NCCN Clinical Practice Guidelines in Oncology (NCCN Guidelines ${ }^{\circledR}$ ) for Head and Neck Cancers V.2.2020. ㅇ 2020 National Comprehensive Cancer Network, Inc. All rights reserved. The NCCN Guidelines ${ }^{\circledR}$ and illustrations herein may not be reproduced in any form for any purpose without the express written permission of NCCN. To view the most recent and complete version of the NCCN Guidelines, go online to NCCN.org. The NCCN Guidelines are a work in progress that may be refined as often as new significant data becomes available. NCCN makes no warranties of any kind whatsoever regarding their content, use or application and disclaims any responsibility for their application or use in any way.

imaging; 4 individuals underwent neck dissection, all of whom had residual disease, and 4 individuals were observed and remained free of disease. Four patients had reduced FDG avidity on 16-week imaging, none of whom had residual disease. Based on these results, the authors recommended that patients with persistent and unchanged FDG avidity on 16-week PET/CT should undergo neck dissection. Given this small number of patients, it is difficult to comment on the appropriate management of patients with residual metabolic activity on repeat PET/CT.

Delayed timing of restaging PET/CT. Delaying the time of restaging PET/CT scan was proposed in a 2014 publication by Vainshtein et al, who evaluated the performance of 12-week PET/CT in predicting local and regional failure after CRT (8). The authors retrospectively reviewed and re-classified post-therapy scans in 101 patients with AJCC 7th edition stage III and IV HPV-associated OPSCC, grouping responses into complete, near complete, and less than complete. The PPV of 12-week PET/CT was found to be $33 \%$ for detection of nodal disease. Given this low PPV, the authors also assessed the diagnostic performance of surveillance PET/CT scans in 67 patients who did not have any clinical or radiographic suspicion of recurrent disease. The median time from completion of CRT to first surveillance PET/CT was 7.3 months. All patients (12) with an incomplete response on restaging PET/CT (near and less than complete) achieved a complete response on surveillance PET/CT. The authors reported that surveillance PET/CT had a NPV of $98 \%$ and PPV of $83 \%$ for detection of regional recurrence. Based on the superior operating characteristics of surveillance PET/CT, the authors suggested that restaging PET/CT could be delayed to 6 months after completion of CRT.

When considering a delay in restaging PET/CT, it is necessary to consider the optimal timing since prolonging assessment could impact patient anxiety, neck dissection complication rates, type of neck dissection (selective versus modified versus radical), regional control, and early detection of distant metastases. In a 2010 retrospective analysis of 105 patients with HNSCC who underwent neck dissection after CRT, Goguen et al compared the complication rates and survival outcomes between patients undergoing neck dissection less than 12 weeks versus greater than 12 weeks after CRT (20). The authors found that the two groups did not differ in the rate of surgical complications or survival. This type of analysis has not been performed for patients with HPV-associated OPSCC. In regards to detection of distant metastases, some studies indicate that they occur at later time 


\section{Approach to the risk-adapted management of node positive HNSCC post RT}

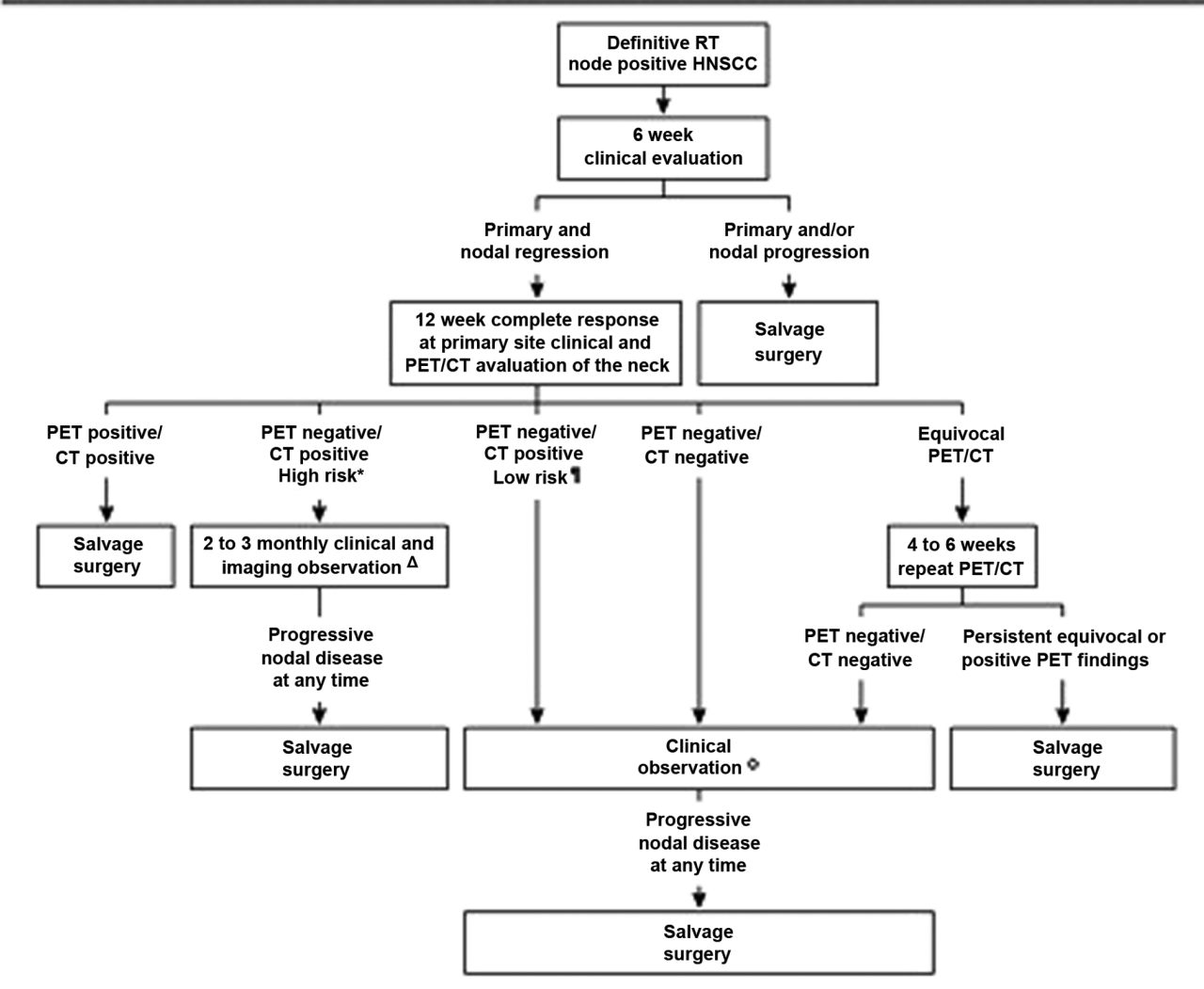

HNSCC: head and neck squamous cell carcinoma; RT: radiation therapy; PET: positron emission tomography; CT: computed tomography; OPSCC: oropharyngeal squamous cell carcinoma; HPV: human papilloma virus. *High risk: where the perceived risk of residual nodal disease is high, such as suboptimally treated patients due to premature cessation of treatment, unplanned treatment interruptions, or the presence of high-risk primary disease, such as non-OPSCC.

ๆLow risk: where the perceived risk of residual nodal disease is low, such as HPV associated OPSCC treated optimally.

$\Delta$ Ongoing clinical and imaging observation until residuum is $<1$ to $1.5 \mathrm{~cm}$ or stable for $>6$ months.

$\diamond$ The frequency of follow-up is based on institutional policy.

Figure 2. UpToDate's Approach to the Risk-adapted Management of Node Positive Head and Neck Squamous Cell Carcinoma Post-Radiation. From 'Management of the neck following definitive radiotherapy with or without chemoradiotherapy in head and neck squamous cell carcinoma.'

points in HPV-associated OPSCC (21). Therefore, the role of early PET/CT for this purpose may not be significant.

Initial assessment with multimodality or alternative imaging modalities. Several publications have highlighted the use of ancillary imaging modalities to assist with treatment assessment, either alone or in combination with PET/CT. In a retrospective study from 2014, Pellini et al investigated the diagnostic performance of a 12-week post-CRT neck ultrasound, MRI, and PET/CT for detection of residual nodal disease (22). The authors prospectively enrolled 36 patients with OPSCC bulky nodal disease $(>3 \mathrm{~cm})$ who were treated with definitive CRT. All patients in the study underwent planned neck dissection 3 months after treatment; the pathology reports were used to determine the operating characteristics of the three imaging modalities. Individuals with less than a complete response at the primary site were excluded. For response evaluation, lymph nodes were considered metastatic on ultrasound if they had a 'short axis diameter $>7 \mathrm{~mm}$ and/or round shape (reduction in the ratio of maximal longitudinal to maximal axial diameter), unclear boundary or irregular hilar and internal echoes.' When comparing each modality alone and in combination, the authors found that neck ultrasound and PET/CT had the highest NPV (93.3\%), while a combination of neck ultrasound, MRI, and FDG-PET/CT had the highest PPV (100\%). The authors suggested that neck ultrasound with FDG-PET/CT could be used to identify patients with a complete response, while a combination of ultrasound, MRI, and FDG-PET/CT could be useful in selecting patients who require neck dissection. Of note, the authors did not include information on HPV or p16 status, limiting the applicability of these findings. Moreover, the cost of obtaining three imaging modalities concurrently is an important consideration; the authors argued that these health care expenditures would be offset by a decreased number of unnecessary surgical procedures. It is critical that further research is performed to investigate the cost-effectiveness of this strategy.

Yu et al compared the diagnostic performance of diffusion-weighted magnetic resonance imaging (DWI-MRI) and 


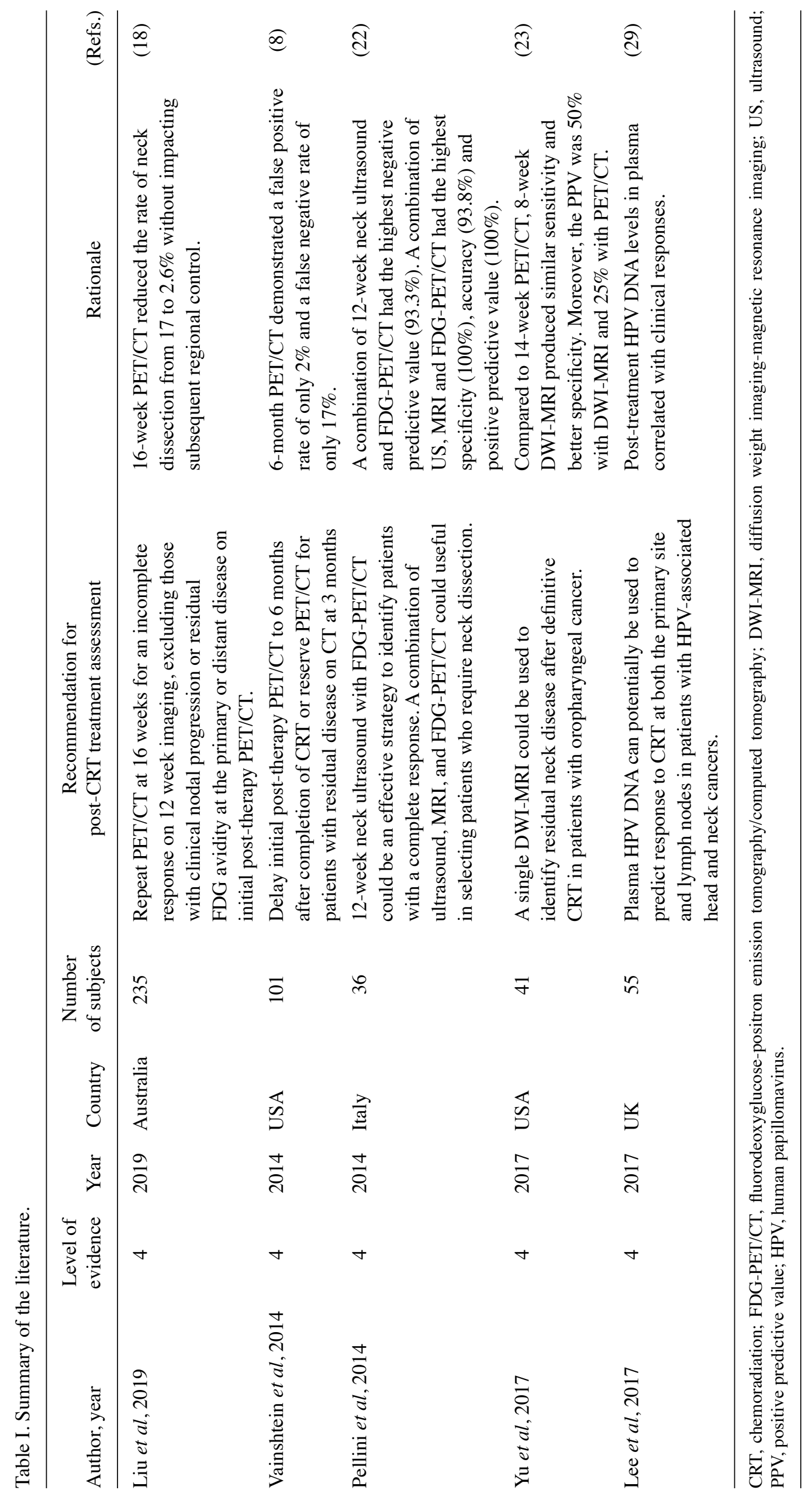


PET/CT for detection of persistent neck disease (23). DWI-MRI is a functional imaging modality that allows for calculation of an apparent diffusion coefficient (ADC), which reflects microscopic proton motion; a lower value is associated with the presence of cancer. The rationale for this modality is that it can detect persistent disease earlier as diffusion signals are evident before changes in tumor size. The authors retrospectively reviewed the records of 41 patients with HPV-associated OPSCC who were treated with definitive CRT. DWI-MRI and PET/CT were performed at a median time of 8.6 and 14 weeks, respectively. The mean ADC and lymph node volume were determined for each DWI-MRI; the maximum standardized uptake value (SUV) was measured for each PET/CT. Using a receiver operating characteristic (ROC) analysis, the authors calculated a threshold of 1,500 for ADC and 2.5 for SUVmax. Using these thresholds and the pathology results of either biopsy or neck dissection, the authors found that DWI-MRI had a NPV and PPV of 100 and 50\%, while PET/CT had a NPV and PPV of 100 and 25\%. Given the earlier and more specific detection of residual nodal disease with DWI-MRI, the authors proposed that this imaging modality could be used to identify residual neck disease after definitive CRT.

This study was not the first to demonstrate the utility of DWI-MRI in head and neck cancer, but was the only publication that limited their analysis to HPV-associated OPSCC. While the higher PPV, equivalent NPV, and earlier time of acquisition support the use of DWI-MRI, it is important to consider the fact that surveillance with PET/CT had shown to be noninferior to planned neck dissection in regards to survival, which may dissuade clinicians from abandoning PET/CT. Further, although the PPV of DWI-MRI was shown to be higher than PET/CT, a value of 50\% may still be suboptimal. Another issue is the ability of DWI-MRI to detect residual disease at the primary site. In a 2017 publication with 46 patients with advanced OPSCC, Greuter et al reported a PPV of $60.0 \%$ for DWI-MRI (24). However, less than half of the cohort had HPV-associated disease. Finally, Yu et al highlighted several important limitations of their study, including a small sample size and retrospective design, as well as limitations of DWI-MRI, such as a complex technique and difficulty in evaluation of cystic nodes (23). Therefore, although DWI-MRI may offer earlier and more specific detection of residual neck disease, which could help limit unnecessary neck dissections, this modality also has limitations.

Detection of circulating HPV DNA. The detection of plasma HPV DNA is an exciting new development that may prove useful in detection of residual disease (25-28). Lee et al assessed the role of circulating HPV DNA in detection of residual disease after CRT (29). The authors prospectively enrolled 88 patients with locally-advanced head and neck cancers and divided them into test (55) and validation (33) cohorts. All participants had HPV-associated disease, determined by p16 immunohistochemistry (IHC) and E6 RT-PCR, and underwent 12 -week PET/CT. Individuals with residual FDG-avid nodes had a neck dissection and those with residual uptake at the primary or distant site underwent biopsy. The authors developed an amplicon-based next generation sequencing (NGS) assay to detect circulating HPV DNA in serum. They collected plasma samples at baseline as well as
6 and 12 weeks post-CRT. To validate the detection assay, the authors compared pre-CRT HPV DNA levels to both p16 IHC and E6 RT-PCR; they reported a sensitivity and specificity of 90\% and above. Moreover, the authors correlated post-treatment HPV DNA levels to PET/CT and pathology reports. In the test cohort, HPV DNA levels were below the threshold of detection in all patients (23) with a complete radiological response on 12-week PET/CT. The assay was positive in the only patient with biopsy-proven persistent disease. Further, HPV DNA was undetectable in 3 patients who underwent biopsy at the primary and 1 patient who underwent neck dissection for increased FDG uptake on 12-week PET/CT; the pathology was negative in all 4 cases. In the validation cohort, HPV DNA levels were below the threshold of detection in all 7 patients with a complete radiographic response. Moreover, the assay was negative in 3 patients who underwent neck dissection for increased FDG on 12-week PET/CT; no residual disease was found in any of these patients. Based on these findings, the authors suggested that plasma HPV DNA could be used to help guide treatment decisions and avoid unnecessary surgeries, but commented that their findings require validation in a larger cohort of patients with longer follow-up. Indeed, the study had a small number of patients with residual disease and/or recurrence. Moreover, it would be helpful to compare the utility of this sequencing-based plasma assay to other methods of HPV detection, such as those that involve salivary collection or PCR technology.

\section{Conclusions and prospects}

This literature review highlights several strategies that have been proposed to improve post-CRT neck assessment in patients with HPV-associated OPSCC. A repeat PET/CT at 16-weeks for patients with an incomplete response on 12 -week imaging may help reduce the number of neck dissections without comprising regional control. Delaying the timing of the initial post-therapy PET/CT may improve its PPV by allowing for radiation-induced inflammation to resolve, but the appropriate timing is unknown and has important clinical implications. DWI-MRI may offer an alternative to PET/CT, but has its own limitations. Finally, detection of plasma HPV DNA is an exciting development that may be able to effectively detect residual disease, but it requires further validation in larger cohorts. Given the current limitations of post-CRT assessment of the neck in patients with HPV-associated OPSCC, further investigation of these strategies will be highly beneficial for this population.

\section{Acknowledgements}

Not applicable.

\section{Funding}

No funding was received.

\section{Availability of data and materials}

Not applicable. 


\section{Authors' contributions}

MW performed the literature review, wrote the manuscript and edited the final version. MG, LM, TT, NS, DKa, DF and DKr reviewed and edited the manuscript. ST reviewed the published literature, wrote parts of the manuscript, and edited and approved the final version. All authors read and approved the final version of the manuscript.

\section{Ethics approval and consent to participate}

Not applicable.

\section{Patient consent for publication}

Not applicable.

\section{Competing interests}

The authors declare that they have no competing interests.

\section{References}

1. Reproduced with permission from the NCCN Clinical Practice Guidelines in Oncology (NCCN Guidelines $\left.{ }^{\circledR}\right)$ for Head and Neck Cancers V.1.2020. (C) 2020 National Comprehensive Cancer Network, Inc. All rights reserved. The NCCN Guidelines ${ }^{\circledR}$ and illustrations herein may not be reproduced in any form for any purpose without the express written permission of NCCN To view the most recent and complete version of the NCCN Guidelines, go online to NCCN.org. The NCCN Guidelines are a work in progress that may be refined as often as new significant data becomes available. NCCN makes no warranties of any kind whatsoever regarding their content, use or application and disclaims any responsibility for their application or use in any way.

2. Porceddu SV and Weber RS: Management of the neck following definitive radiotherapy with or without chemoradiotherapy in head and neck squamous cell carcinoma. Post TW, ed. UpToDate. Waltham, MA, 2018.

3. Mehanna H, Wong WL, McConkey CC, Rahman JK, Robinson M, Hartley AG, Nutting C, Powell N, Al-Booz H, Robinson M, et al: PET-CT surveillance versus neck dissection in advanced head and neck cancer. N Engl J Med 374: 1444-1454, 2016.

4. Garden AS, Gunn GB, Hessel A, Beadle BM, Ahmed S, El-Naggar AK, Fuller CD, Byers LA, Phan J, Frank SJ, et al: Management of the lymph node-positive neck in the patient with human papillomavirus-associated oropharyngeal cancer. Cancer 120: 3082-3088, 2014.

5. Goenka A, Morris LG, Rao SS, Wolden SL, Wong RJ, Kraus DH, Ohri N, Setton J, Lok BH, Riaz N, et al: Long-term regional control in the observed neck following definitive chemoradiation for node-positive oropharyngeal squamous cell cancer. Int J Cancer 133: 1214-1221, 2013.

6. Koshkareva Y, Branstetter BF, Gaughan JP and Ferris RL: Predictive accuracy of first post-treatment PET/CT in HPV-related oropharyngeal squamous cell carcinoma. Laryngoscope 124 $1843-1847,2014$

7. Chan JY, Sanguineti G, Richmon JD, Marur S, Gourin CG, Koch W, Chung CH, Quon H, Bishop JA, Aygun N and Agrawal N: Retrospective review of positron emission tomography with contrast-enhanced computed tomography in the posttreatment setting in human papillomavirus-associated oropharyngeal carcinoma. Arch Otolaryngol Head Neck Surg 138: 1040-1046, 2012.

8. Vainshtein JM, Spector ME, Stenmark MH, Bradford CR, Wolf GT, Worden FP, Chepeha DB, McHugh JB, Carey T, Wong KK and Eisbruch A: Reliability of post-chemoradiotherapy F-18-FDG PET/CT for prediction of locoregional failure in human papillomavirus-associated oropharyngeal cancer. Oral Oncol 50: 234-239, 2014.
9. Wang K, Wong TZ, Amdur RJ, Mendenhall WM, Sheets NC, Green R, Thorp BD, Patel SN, Hackman TG, Zanation AM, et al: Pitfalls of post-treatment PET after de-intensified chemoradiotherapy for HPV-associated oropharynx cancer: Secondary analysis of a phase 2 trial. Oral Oncol 78: 108-113, 2018.

10. Helsen N, Van den Wyngaert T, Carp L and Stroobants S: FDG-PET/CT for treatment response assessment in head and neck squamous cell carcinoma: A systematic review and meta-analysis of diagnostic performance. Eur J Nucl Med Mol Imaging 45: 1063-1071, 2018.

11. Taghipour M, Marcus C, Califano J, Fakhry $\mathrm{C}$ and Subramaniam RM: The value of follow-up FDG-PET/CT in the management and prognosis of patients with HPV-positive oropharyngeal squamous cell carcinoma. J Med Imaging Radiat Oncol 59: 681-686, 2015.

12. Shonka DC Jr, Shoushtari AN, Thomas CY, Moskaluk C, Read PW, Reibel JF, Levine PA and Jameson MJ: Predicting residual neck disease in patients with oropharyngeal squamous cell carcinoma treated with radiation therapy: Utility of p16 status. Arch Otolaryngol Head Neck Surg 135: 1126-1132, 2009.

13. Corpman DW, Masroor F, Carpenter DM, Nayak S, Gurushanthaiah D and Wang KH: Posttreatment surveillance PET/CT for HPV-associated oropharyngeal cancer. Head Neck 41: 456-462, 2019.

14. Huang SH, O'Sullivan B, Xu W, Zhao H, Chen DD, Ringash J, Hope A, Razak A, Gilbert R, Irish J, et al: Temporal nodal regression and regional control after primary radiation therapy for N2-N3 head-and-neck cancer stratified by HPV status. Int J Radiat Oncol Biol Phys 87: 1078-1085, 2013.

15. Van der Putten L, Van den Broek GB, De Bree R, van den Brekel MW, Balm AJ, Hoebers FJ, Doornaert P, Leemans CR and Rasch CR: Effectiveness of salvage selective and modified radical neck dissection for regional pathologic lymphadenopathy after chemoradiation. Head Neck 31: 593-603, 2009.

16. Fleischman GM, Thorp BD, Difurio $M$ and Hackman TG: Accuracy of ultrasonography-guided fine-needle aspiration in detecting persistent nodal disease after chemoradiotherapy. JAMA Otolaryngol Head Neck Surg 142: 377-382, 2016.

17. Kao SS and Ooi EH: Survival outcomes following salvage surgery for oropharyngeal squamous cell carcinoma: Systematic review. J Laryngol Otol 132: 299-313, 2018.

18. Liu HY, Milne R, Lock G, Panizza BJ, Bernard A, Foote M, McGrath M, Brown E, Gandhi M and Porceddu SV: Utility of a repeat PET/CT scan in HPV-associated oropharyngeal cancer following incomplete nodal response from (chemo)radiotherapy. Oral Oncol 88: 153-159, 2019.

19. Bird T, Barrington S, Thavaraj S, Jeannon JP, Lyons A, Oakley R, Simo R, Lei M and Guerrero Urbano T: (18)F-FDG PET/CT to assess response and guide risk-stratified follow-up after chemoradiotherapy for oropharyngeal squamous cell carcinoma. Eur J Nucl Med Mol Imaging 43: 1239-1247, 2016.

20. Goguen LA, Chapuy CI, Li Y, Zhao SD and Annino DJ: Neck dissection after chemoradiotherapy: Timing and complications. Arch Otolaryngol Head Neck Surg 136: 1071-1077, 2010.

21. Subramaniam RM, Alluri KC, Tahari AK, Aygun N and Quon H: PET/CT imaging and human papilloma virus-positive oropharyngeal squamous cell cancer: Evolving clinical imaging paradigm. J Nucl Med 55: 431-438, 2014.

22. Pellini R, Manciocco V, Turri-zanoni M, Vidiri A, Sanguineti G, Marucci L, Sciuto R, Covello R, Sperduti I, Kayal R, et al: Planned neck dissection after chemoradiotherapy in advanced oropharyngeal squamous cell cancer: The role of US, MRI and FDG-PET/TC scans to assess residual neck disease. J Craniomaxillofac Surg 42: 1834-1839, 2014.

23. Yu Y, Mabray M, Silveira W, Shen PY, Ryan WR, Uzelac A and Yom SS: Earlier and more specific detection of persistent neck disease with diffusion-weighted MRI versus subsequent PET/CT after definitive chemoradiation for oropharyngeal squamous cell carcinoma. Head Neck 39: 432-438, 2017.

24. Greuter MJ, Schouten CS, Castelijns JA, de Graaf P, Comans EF, Hoekstra OS, de Bree R and Coupé VM: Cost-effectiveness of response evaluation after chemoradiation in patients with advanced oropharyngeal cancer using F-FDG-PET-CT and/or diffusion-weighted MRI. BMC Cancer 17: 256, 2017.

25. Gupta GP, Kumar S, Marron D, Amdur RJ, Hayes DN, Weiss J, Grilley-Olson J, Zanation A, Hackman T, Zevallos JP, et al: Circulating tumor HPV16 DNA as a biomarker of tumor genomics and disease control in HPV-associated oropharyngeal squamous cell carcinoma. Int J Radiat Oncol Biol Phys 100: 1310-1311, 2018 
26. Ahn SM, Chan JY, Zhang Z, Wang H, Khan Z, Bishop JA, Westra W, Koch WM and Califano JA: Saliva and plasma quantitative polymerase chain reaction-based detection and surveillance of human papillomavirus-related head and neck cancer. JAMA Otolaryngol Head Neck Surg 140: 846-854, 2014

27. Dahlstrom KR, Li G, Hussey CS, Vo JT, Wei Q, Zhao C and Sturgis EM: Circulating human papillomavirus DNA as a marker for disease extent and recurrence among patients with oropharyngeal cancer. Cancer 121: 3455-3464, 2015.

28. Jensen KK, Grønhøj C, Jensen DH and Von buchwald C: Circulating human papillomavirus DNA as a surveillance tool in head and neck squamous cell carcinoma: A systematic review and meta-analysis. Clin Otolaryngol 43: 1242-1249, 2018.
29. Lee JY, Garcia-Murillas I, Cutts RJ, De Castro DG, Grove L, Hurley T, Wang F, Nutting C, Newbold K, Harrington K, et al: Predicting response to radical (chemo)radiotherapy with circulating HPV DNA in locally advanced head and neck squamous carcinoma. Br J Cancer 117: 876-883, 2017. 